Chinese Journal of Organic Chemistry

\title{
钯催化分子内胺化合成苯并噁嗪酮衍生物
}

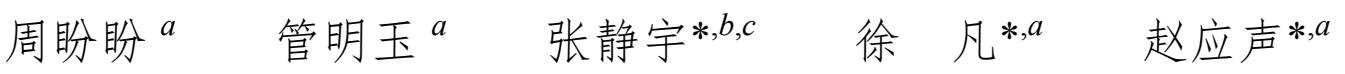 \\ ( ${ }^{a}$ 苏州大学材料化学化工学部 苏州 215123) \\ ( ${ }^{b}$ 苏州大学物理与光电学部 能源与材料创新研究院 苏州 215123) \\ ( ${ }^{c}$ 江苏省先进碳材料与可穿戴能源技术重点实验室 苏州 215123)
}

\begin{abstract}
摘要 苯并噁嗪酮及其衍生物具有重要的医用价值及生理活性，因此合成该类骨架结构受到很多化学家的重视. 报道 了一种以含 $\mathrm{N}, \mathrm{O}$-双齿导向基团的苯氧乙酸为底物, 通过钯催化的 $\mathrm{C}-\mathrm{H}$ 键活化反应一步构建 $\mathrm{C}-\mathrm{N}$ 键的新方法, 合成 了一系列苯并噁嗪类化合物. 该方法反应条件温和，操作简便，合成效率高.
\end{abstract}

关键词 $\mathrm{C}-\mathrm{H}$ 键活化; 钯催化; 苯氧乙酸; 分子内 $\mathrm{C}-\mathrm{N}$ 成键

\section{Facile Synthesis of Benzoxazinone Derivatives via Palladium Catalyzed Intramolecular Amination}

Zhou, Panpan ${ }^{a} \quad$ Guan, Mingyu $^{a} \quad$ Zhang, Jingyu ${ }^{*, b, c} \quad$ Xu, Fan ${ }^{*, a} \quad$ Zhao, Yingsheng ${ }^{*, a}$

$\left({ }^{a}\right.$ College of Chemistry, Chemical Engineering and Materials Science, Soochow University, Suzhou 215123)

$\left({ }^{b}\right.$ Soochow Institute for Energy and Materials Innovation, College of Physics, Optoelectronics and Energy \& Collaborative Innovation Center of Suzhou Nano Science and Technology, Soochow University, Suzhou 215123)

( ${ }^{c}$ Key Laboratory of Advanced Carbon Materials and Wearable Energy Technologies of Jiangsu Province, Suzhou 215123)

\begin{abstract}
Benzoxazinone and its derivatives are well-known as a broad-spectrum of physiological activities compounds. Herein we report a novel approach for the synthesis of benzoxazine derivatives via a $N, O$-bindentate directing assisted Pd-catalyzed intramolecular amination reactions. Various benzoxazinone derivatives are obtained in moderate to good yields. The directing group can be removed under mild condition, highlighting potential synthetic utility of this method.

Keywords C - H functionalization; palladium catalysis; phenoxyacetic acid; intramolecular amination
\end{abstract}

苯并噁嗪酮及其衍生物具有广泛的生物活性，在农

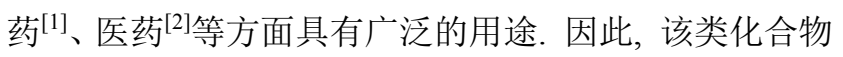
受到人们广泛的关注, 近年来化学家们已合成了不少具 有苯并噁坐酮骨架的生物活性物质. 例如, 化合物 I 是 抗菌药物 ${ }^{[3]}$, 化合物 II 是很好的杀虫剂 ${ }^{[4]}$, 化合物 III 是 大豆、花生常用的除草剂 ${ }^{[5]}$ (图 1).

1944 年, Holly 和 Cope ${ }^{[6]}$ 首次在 Mannich 反应中意 外地发现了 $2 H-1,3$-苯并噁嗪化合物, 随后这类物质便 广泛用于合成高分子树脂 ${ }^{[7]} .1962$ 年, Hamilton ${ }^{[8]}$ 发现含 羟胺酸结构的 $2 H-1,3$-苯并噁嗪类化合物具有生物活性, 开启了该类物质通向农药、医药方向的大门. 合成苯并<smiles>CN1C(=O)COc2ccc(C(O)Cn3ccnc3)cc21</smiles><smiles>COc1ccc2c(c1)OC(O)C(=O)N2O[Mg]</smiles><smiles>C#CCN1C(=O)COc2cc(F)c(N3C(=O)C4=C(CCCC4)C3=O)cc21</smiles>

图 1 含有苯并噁嗪酮骨架的药物

Figure 1 Selected drugs containing benzoxazinone skeletons 啞嗪化合物的主要方法有生物合成法 ${ }^{[9]}$ 和化学合成法.

* Corresponding author. E-mail: yszhao@suda.edu.cn Received March 6, 2017; revised April 8, 2017; published online April 21, 2017.

Project supported by the National Natural Science Foundation of China (No. 21572149) and the Young National Natural Science Foundation of China (Nos. 21402133, 21403148).

国家自然科学基金(No. 21572149)和国家自然科学青年基金(Nos. 21402133, 21403148)资助项目. 
其中, 化学合成法主要是通过邻硝基酚 ${ }^{[10]}$ 、邻氨基 酚 $^{[11] \text { 、邻氯酚 }}{ }^{[12]}$ 或邻碘酚 ${ }^{[13]}$ 来合成苯并噁嗪化合物.

通过文献调研, 我们发现通过 $\mathrm{C}-\mathrm{H}$ 键活化来合成 该类化合物的方法还未见诸报道. $\mathrm{C}-\mathrm{H}$ 键活化反应具 有原子经济性的优势, 符合绿色化学的原则 ${ }^{[14]}$. $\mathrm{C}-\mathrm{H}$ 键活化反应多数需要导向基团, 其中, 基于羧酸类底物 的导向基团近年来受到广泛研究，例如 8-氨基喹啉 ${ }^{[15]}$ 、 氨基酯 ${ }^{[16]}$ 、甲氧基胺 ${ }^{[17]}$ 等. 近期, 我们课题组构建了一 种 $N, N$-二甲基甘氨酰胺导向基, 并将其成功运用于羧酸 类底物的 $\mathrm{C}-\mathrm{H}$ 键活化反应 ${ }^{[18]}$.

在此, 我们成功利用 $N, N$-二甲基甘氨酰胺导向基, 通过 $\mathrm{C}-\mathrm{H}$ 键活化反应, 实现了苯氧乙酸类化合物分子 内 $\mathrm{C}-\mathrm{N}$ 成键关环反应，以中等至优秀的收率合成了一 系列苯并啞嗪酮衍生物. 该反应条件温和, 易于操作, 底物适用性好。

\section{1 结果与讨论}

\section{1 反应条件的优化}

我们以 $1 \mathrm{a}$ 为模板底物对条件进行优化, 考察了催 化剂、氧化剂、添加剂、溶剂及温度对反应的影响, 实 验结果列于表 1 . 此反应在氩气条件下效果较好, 可能 原因是氩气氛围有利于抑制醋酸化副产物的生成. 由表 1 可知, 醋酸碘苯为氧化剂时效果最好(表 1, Entry 2), 其它氧化剂如氧化银、醋酸银、过硫酸钾等(表 1 , Entries $22 \sim 25$ ) 效果均不好, 而加入酸或碱添加剂则使产率大 幅下降(表 1, Entries 20,21). 通过对溶剂进行篮选(表 1, Entries 9 17), 我们发现六氟异丙醇(HFIP)是该反应的 最优溶剂, 而在二氯甲烷(DCE)、1,4-二氧六环(dioxane)

\section{表 1 反应条件的优化 ${ }^{a}$}

Table 1 Optimization of reaction conditions

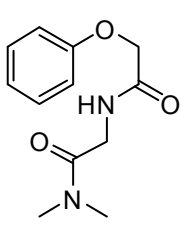

$1 \mathrm{a}$

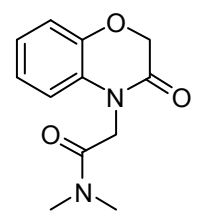

$2 a$

\begin{tabular}{|c|c|c|c|c|c|}
\hline Entry & Catalyst (mmol) & Oxidant & Solvent (mL) & Temperture $/{ }^{\circ} \mathrm{C}$ & $\mathrm{Yield}^{b} / \%$ \\
\hline 1 & - & $\mathrm{PhI}(\mathrm{OAc})_{2}$ & HFIP (2) & 80 & 0 \\
\hline 2 & $\mathrm{Pd}(\mathrm{OAc})_{2}(0.01)$ & $\mathrm{PhI}(\mathrm{OAc})_{2}$ & HFIP (2) & 80 & 87 \\
\hline 3 & $\mathrm{Pd}(\mathrm{OAc})_{2}(0.005)$ & $\mathrm{PhI}(\mathrm{OAc})_{2}$ & HFIP (2) & 80 & 63 \\
\hline 4 & $\mathrm{Cu}(\mathrm{OAc})_{2}(0.01)$ & $\mathrm{PhI}(\mathrm{OAc})_{2}$ & HFIP (2) & 80 & 0 \\
\hline 5 & {$\left[\mathrm{RuCl}_{2}(p \text {-cymene })\right]_{2}(0.01)$} & $\mathrm{PhI}(\mathrm{OAc})_{2}$ & HFIP (2) & 80 & 0 \\
\hline 6 & {$\left[\mathrm{Cp}^{*} \mathrm{IrCl}_{2}\right]_{2}(0.01)$} & $\mathrm{PhI}(\mathrm{OAc})_{2}$ & HFIP (2) & 80 & 0 \\
\hline 7 & $\mathrm{Pd}(\mathrm{OAc})_{2}(0.01)$ & $\mathrm{PhI}(\mathrm{OAc})_{2}$ & HFIP (1) & 80 & 55 \\
\hline 8 & $\mathrm{Pd}(\mathrm{OAc})_{2}(0.01)$ & $\mathrm{PhI}(\mathrm{OAc})_{2}$ & HFIP $(0.5)$ & 80 & 36 \\
\hline 9 & $\mathrm{Pd}(\mathrm{OAc})_{2}(0.01)$ & $\mathrm{PhI}(\mathrm{OAc})_{2}$ & Toluene (2) & 80 & 14 \\
\hline 10 & $\mathrm{Pd}(\mathrm{OAc})_{2}(0.01)$ & $\mathrm{PhI}(\mathrm{OAc})_{2}$ & $\operatorname{DCE}(2)$ & 80 & 18 \\
\hline 11 & $\mathrm{Pd}(\mathrm{OAc})_{2}(0.01)$ & $\mathrm{PhI}(\mathrm{OAc})_{2}$ & Dioxane (2) & 80 & 17 \\
\hline 12 & $\mathrm{Pd}(\mathrm{OAc})_{2}(0.01)$ & $\mathrm{PhI}(\mathrm{OAc})_{2}$ & $\mathrm{MeCN}(2)$ & 80 & 0 \\
\hline 14 & $\mathrm{Pd}(\mathrm{OAc})_{2}(0.01)$ & $\mathrm{PhI}(\mathrm{OAc})_{2}$ & THF (2) & 80 & 0 \\
\hline 15 & $\mathrm{Pd}(\mathrm{OAc})_{2}(0.01)$ & $\mathrm{PhI}(\mathrm{OAc})_{2}$ & t-AmylOH (2) & 80 & 60 \\
\hline 16 & $\mathrm{Pd}(\mathrm{OAc})_{2}(0.01)$ & $\mathrm{PhI}(\mathrm{OAc})_{2}$ & DMSO (2) & 80 & 0 \\
\hline 17 & $\mathrm{Pd}(\mathrm{OAc})_{2}(0.01)$ & $\mathrm{PhI}(\mathrm{OAc})_{2}$ & $\mathrm{PhCl}(2)$ & 80 & 37 \\
\hline 18 & $\mathrm{Pd}(\mathrm{OAc})_{2}(0.01)$ & $\mathrm{PhI}(\mathrm{OAc})_{2}$ & HFIP (2) & 60 & 50 \\
\hline 19 & $\mathrm{Pd}(\mathrm{OAc})_{2}(0.01)$ & $\mathrm{PhI}(\mathrm{OAc})_{2}$ & HFIP (2) & 100 & 76 \\
\hline $20^{c}$ & $\mathrm{Pd}(\mathrm{OAc})_{2}(0.01)$ & $\mathrm{PhI}(\mathrm{OAc})_{2}$ & HFIP (2) & 80 & 67 \\
\hline $21^{d}$ & $\mathrm{Pd}(\mathrm{OAc})_{2}(0.01)$ & $\mathrm{PhI}(\mathrm{OAc})_{2}$ & HFIP (2) & 80 & 60 \\
\hline 22 & $\mathrm{Pd}(\mathrm{OAc})_{2}(0.01)$ & $\mathrm{K}_{2} \mathrm{~S}_{2} \mathrm{O}_{8}$ & HFIP (2) & 80 & 0 \\
\hline 23 & $\mathrm{Pd}(\mathrm{OAc})_{2}(0.01)$ & AgOAc & HFIP (2) & 80 & 0 \\
\hline 24 & $\mathrm{Pd}(\mathrm{OAc})_{2}(0.01)$ & $\mathrm{Ag}_{2} \mathrm{O}$ & HFIP (2) & 80 & 0 \\
\hline 25 & $\mathrm{Pd}(\mathrm{OAc})_{2}(0.01)$ & NFSI & HFIP (2) & 80 & 0 \\
\hline
\end{tabular}

${ }^{a}$ Conditions: 1a $(0.1 \mathrm{mmol})$, catalyst, oxidant $(0.2 \mathrm{mmol})$, solvent, argon $(101 \mathrm{kPa}), 24 \mathrm{~h}$. Yields were based on GC analysis using $n$-tridecaneas an internal standard; ${ }^{b}$ Isolated yield; ${ }^{c} \mathrm{~K}_{2} \mathrm{CO}_{3}(0.2 \mathrm{mmol})$ was added; ${ }^{d} \mathrm{PivOH}(0.03 \mathrm{mmol})$ was added. 
或甲苯(toluene)中效果不理想, 而在其它常用溶剂如四 氢呋喃(THF)、 $N, N$-二甲基甲酰胺(DMF)、乙腈 $(\mathrm{MeCN})$ 中反应几乎不能进行. 在随后对溶剂量的笁选中我们发 现, 当溶剂量为 0.5 或 $1 \mathrm{~mL}$ 时, 产率都急剧下降, 因此 我们选用溶剂量为 $2 \mathrm{~mL}$ (表 1, Entries 7,8). 在对温度进 行篮选时(表 1, Entries 18, 19), 我们发现温度对反应的 影响不是很大, 在 $80{ }^{\circ} \mathrm{C}$ 时效果最好. 最后, 我们尝试 降低催化剂的用量(表 1, Entry 3), 然而当催化剂的用量 降下来时, 反应效果变差. 值得一提的是, 如果体系中 不添加催化剂, 或使用其他种类的金属催化剂时, 该反 应不能发生(表 1, Entries 1, 4 6). 通过以上条件的优 化, 我们得出了模板反应的最优条件, 即当反应底物为 $0.1 \mathrm{mmol}$ 时, 加入 $0.01 \mathrm{mmol}$ 的 $\mathrm{Pd}(\mathrm{OAc})_{2}$ 作催化剂, 2 $\mathrm{mL}$ 六氟异丙醇(HFIP)作溶剂, $0.2 \mathrm{mmol}$ 醋酸碘苯作氧化 剂, 氩气氛围, 在 $80{ }^{\circ} \mathrm{C}$ 下反应 $24 \mathrm{~h}$, 即可以高收率得到 分子内关环产物.

\section{2 底物的拓展}

在上述最佳的反应条件下, 我们对该反应体系的底 物适用性进行了考察, 结果列于表 2 , 我们发现当苯环 上是卤素取代时, 能够以 $60 \% \sim 95 \%$ 的分离收率得到目 标产物(表 2, Entries 2, 8, 10,11,13). 当苯环上是供电子 基团取代时，我们也能取得中等至优秀的产率(表 2 , Entries 5, 7, 9). 当苯环上连有吸电子基团时, 我们分别 能以 $47 \%$ 和 $75 \%$ 的分离收率得到目标产物 $2 \mathrm{c}$ 和 $2 \mathrm{~d}$ (表 2, Entries 3, 4). 多数底物都有较高的收率, 但当苯氧乙 酸类底物对位是甲氧基时(表 2, Entry 7), 生成的产物主 要是关环醋酸化产物 $2 \mathrm{~g}$. 当以 $1 \mathrm{n}$ 为反应底物时, 我们 能够以 $49 \%$ 的分离收率获得目标产物(表 2, Entry 14).

\section{3 克级放大反应}

随后, 我们在标准条件下进行了克级反应, 仅需使 用 $5 \mathrm{~mol} \%$ 的催化剂量反应 $24 \mathrm{~h}$, 就能够以 $61 \%$ 的分离 收率得到目标产物 2a (Eq. 1), 当反应时间延长至 $48 \mathrm{~h}$ 时, 收率可达到 $73 \%$.

\section{4 导向基的切断}

通常来说，一个好的导向基应具备合成方便，移除 亦简便的特点. 所以我们对我们构建的导向基进行了移 除的研究. 我们发现, 在浓盐酸回流的条件下我们可以 将导向基切除, 以 54\%的分离收率得到脱除导向基团的 产物 3a (Eq. 2), 其氨基可以根据需要进行进一步修饰 和转化.

\section{5 可能的反应机理}

基于实验结果及已有文献[19]的报道，我们提出了 可能的反应机理(Scheme 1). 以底物 $\mathbf{1 a}$ 为例, 首先, 醋
表 2 钯催化合成苯并啞嗪酮 2

Table 2 Palladium catalyzed synthesis of benzoxazinone 2
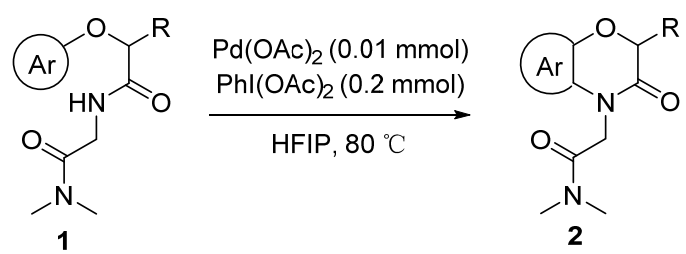

\begin{tabular}{clccc}
\hline Entry & $\mathrm{Ar}$ & $\mathrm{R}$ & Product & Yield/\% \\
\hline 1 & $\mathrm{Ph}$ & $\mathrm{H}$ & $\mathbf{2 a}$ & 87 \\
2 & $o-\mathrm{ClC}_{6} \mathrm{H}_{4}$ & $\mathrm{H}$ & $\mathbf{2 b}$ & 91 \\
3 & $o-\mathrm{O}_{2} \mathrm{NC}_{6} \mathrm{H}_{4}$ & $\mathrm{H}$ & $\mathbf{2 c}$ & 47 \\
4 & $o-\mathrm{HOCC}_{6} \mathrm{H}_{4}$ & $\mathrm{H}$ & $\mathbf{2 d}$ & 75 \\
5 & $o-\mathrm{CH}_{3} \mathrm{OC}_{6} \mathrm{H}_{4}$ & $\mathrm{H}$ & $\mathbf{2 e}$ & 67 \\
6 & $p-\mathrm{AcOC}_{6} \mathrm{H}_{4}$ & $\mathrm{H}$ & $\mathbf{2 f}$ & 70 \\
7 & $p-\mathrm{CH}_{3} \mathrm{OC}_{6} \mathrm{H}_{4}$ & $\mathrm{H}$ & $\mathbf{2 g}$ & 52 \\
8 & $p-\mathrm{FC}_{6} \mathrm{H}_{4}$ & $\mathrm{H}$ & $\mathbf{2 h}$ & 78 \\
9 & $p-\mathrm{CH}_{3} \mathrm{C}_{6} \mathrm{H}_{4}$ & $\mathrm{H}$ & $\mathbf{2 i}$ & 82 \\
10 & $m-\mathrm{ClC}_{6} \mathrm{H}_{4}$ & $\mathrm{H}$ & $\mathbf{2 j}$ & 60 \\
11 & $m-\mathrm{ClC}_{6} \mathrm{H}_{4}$ & $\mathrm{CH}$ & $\mathbf{2 k}$ & 95 \\
12 & $\mathrm{Ph}$ & $\mathrm{CH}$ & $\mathbf{2 l}$ & 72 \\
13 & $p-\mathrm{Cl}-o-\mathrm{CH}_{3} \mathrm{C}_{6} \mathrm{H}_{3}$ & $\mathrm{H}$ & $\mathbf{2 m}$ & 92 \\
14 & $2-\mathrm{Naphthyl}$ & $\mathrm{H}$ & $\mathbf{2 n}$ & 49 \\
\hline
\end{tabular}

Conditions: $1(0.1 \mathrm{mmol})$, catalyst $(0.01 \mathrm{mmol})$, oxidant $(0.2 \mathrm{mmol})$, solvent $(2$ $\mathrm{mL}), 80{ }^{\circ} \mathrm{C}$, argon $(101 \mathrm{kPa}), 24 \mathrm{~h}$.

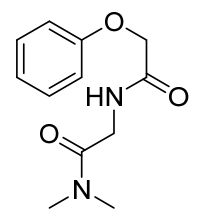

$1 \mathrm{a}(1.18 \mathrm{~g}, 5 \mathrm{mmol})$
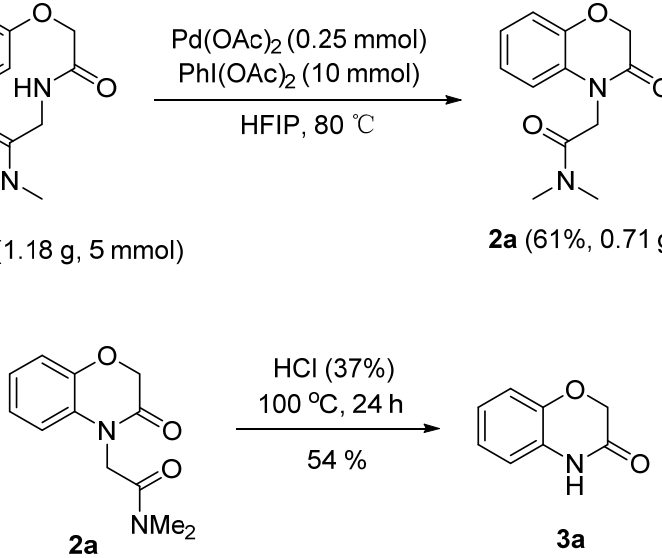

$3 a$

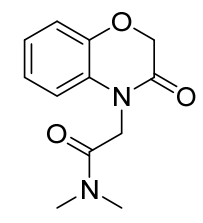

2a $(61 \%, 0.71 \mathrm{~g})$
酸钯和导向基配位, 生成中间体 $\mathbf{A}$, 然后对苯环上的 $\mathrm{C}$ $-\mathrm{H}$ 键进行活化, 形成含钯的七元环过渡态 $\mathbf{B}$, 随后 $\mathbf{B}$ 与醋酸碘苯、进行氧化加成生成中间体 $\mathbf{C}$, 最后发生还 原消除生成目标产物 $\mathbf{2 a}$, 完成催化循环.

\section{2 结论}

综上所述, 通过钯催化的 $\mathrm{C}-\mathrm{H}$ 键活化反应, 实现 了一系列苯并噁嗪衍生物的合成. 该合成方法操作简 单、原子经济、既为苯并噁嗪酮骨架的合成提供了新的 方法，也拓展了我们 $N, N$-二甲基甘氨酰胺导向基的应 用, 同时为构建 $\mathrm{C}-\mathrm{N}$ 成键提供了一条新的途径. 


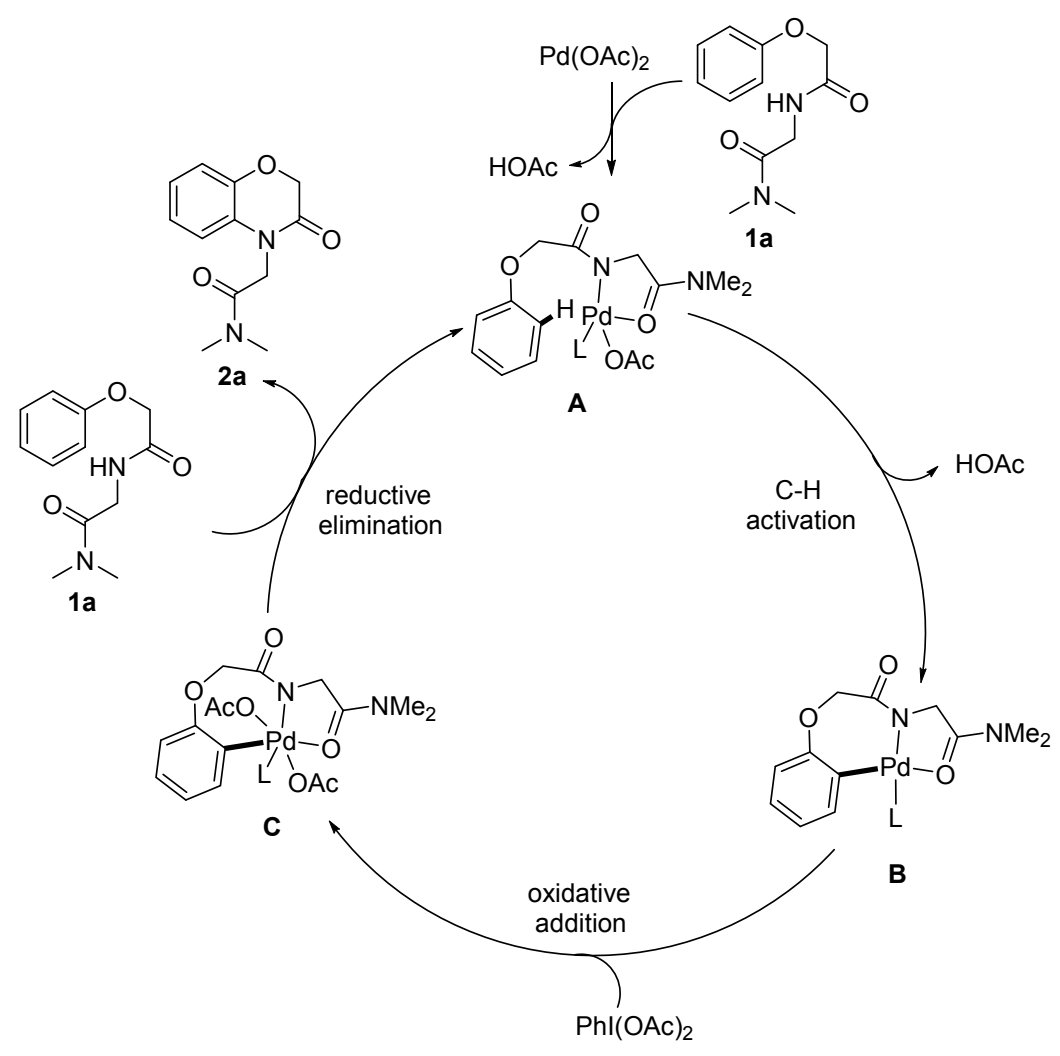

图式 1 可能的反应机理

Scheme 1 Proposed mechanism

\section{3 实验部分}

\section{1 仪器与试剂}

核磁共振氢谱和核磁共振碳谱在 Bruker Avance III $\mathrm{HD}-400 \mathrm{MHz}$ 核磁共振仪上测定, $\mathrm{CDCl}_{3}$ 为溶剂, $\mathrm{TMS}$ 为 内标; 高分辨质谱在 Bruker MicrOTOF-QII 上测定, 气 相色谱在 Thermo Trace 1300 上测定, 熔点在 INESA 仪 电物光熔点仪上测定. 所用试剂除特别说明, 均来自 Acros, Alfa, Adamas, 均为分析纯或化学纯, 使用前未 经纯化. 柱层析所用硅胶为 300 400 目.

\section{2 实验方法}

\section{2 .1 化合物 2 的合成方法}

在 $15 \mathrm{~mL}$ 干燥的螺口反应管中加入化合物 1 (0.1 $\mathrm{mmol}) 、 \mathrm{Pd}(\mathrm{OAc})_{2}(0.01 \mathrm{mmol})$ 、醋酸碘苯 $(0.2 \mathrm{mmol})$ 及 2 $\mathrm{mL}$ HFIP, 混合均匀, 反应管中通入氩气, 后放入 $80{ }^{\circ} \mathrm{C}$ 油浴中搅拌反应 $24 \mathrm{~h}$. 待反应结束后, 将反应体系冷却 到室温, 用旋转蒸发仪除去溶剂, 残余物以 $V($ 石油 醚) $: V($ 乙酸乙酯 $)=1: 3$ 为淋洗剂进行柱层析分离得纯 化合物 2.

$N, N$-二甲基-2-(2H-1,4-苯并噁嗪-3-酮)乙酰胺(2a): 白色固体, 产率 87\%. m.p. 93.1 94.2 ${ }^{\circ} \mathrm{C} ;{ }^{1} \mathrm{H}$ NMR $\left(400 \mathrm{MHz}, \mathrm{CDCl}_{3}\right) \delta: 7.02 \sim 6.95(\mathrm{~m}, 3 \mathrm{H}), 6.79 \sim 6.72(\mathrm{~m}$,
1H), 4.68 (s, 2H), 4.67 (s, 2H), 3.13 (s, 3H), $3.00(\mathrm{~s}, 3 \mathrm{H})$. ${ }^{13} \mathrm{C} \mathrm{NMR}\left(100 \mathrm{MHz}, \mathrm{CDCl}_{3}\right) \delta: 165.4,164.8,144.7,128.6$, 123.5, 122.4, 116.4, 114.5, 67.1, 42.7, 35.9, 35.4. HRMS calcd for $\mathrm{C}_{12} \mathrm{H}_{14} \mathrm{~N}_{2} \mathrm{O}_{3} \mathrm{Na}\left[\mathrm{M}+\mathrm{Na}^{+}\right]$257.0902; found 257.0913 .

$N, N$-二甲基-2-(8-氯-2 $H$-1,4-苯并噁嗪-3-酮) 乙酰 胺(2b): 白色固体，产率 91\%. m.p. 139.9 142.2 ${ }^{\circ} \mathrm{C}$; ${ }^{1} \mathrm{H} \mathrm{NMR}\left(400 \mathrm{MHz}, \mathrm{CDCl}_{3}\right) \delta: 7.07$ (dd, $J=8.1,1.3 \mathrm{~Hz}$, $1 \mathrm{H}), 6.91(\mathrm{t}, J=8.2 \mathrm{~Hz}, 1 \mathrm{H}), 6.66(\mathrm{dd}, J=8.2,1.2 \mathrm{~Hz}$, $1 \mathrm{H}), 4.77$ (s, 2H), 4.68 (s, 2H), 3.13 (s, 3H), 3.01 (s, 3H); ${ }^{13} \mathrm{C}$ NMR $\left(100 \mathrm{MHz}, \mathrm{CDCl}_{3}\right) \delta: 165.1,164.2,140.9,129.9$, 124.3, 122.4, 121.8, 113.0, 67.2, 42.9, 35.9, 35.4. HRMS calcd for $\mathrm{C}_{12} \mathrm{H}_{13} \mathrm{ClN}_{2} \mathrm{O}_{3} \mathrm{Na}\left[\mathrm{M}+\mathrm{Na}^{+}\right]$291.0512; found 291.0516.

$\mathrm{N}, \mathrm{N}$-二甲基-2-(8-硝基-2H-1,4-苯并噁嗪-3-酮)乙 酰胺 (2c): 淡黄色固体，产率 47\%. m.p. 205.9 $208.7{ }^{\circ} \mathrm{C} ;{ }^{1} \mathrm{H}$ NMR $\left(400 \mathrm{MHz}, \mathrm{CDCl}_{3}\right) \delta: 7.63$ (dd, $J=8.2$, $1.4 \mathrm{~Hz}, 1 \mathrm{H}), 7.13(\mathrm{t}, J=8.2 \mathrm{~Hz}, 1 \mathrm{H}), 7.04(\mathrm{dd}, J=8.2,1.4$ $\mathrm{Hz}, 1 \mathrm{H}), 4.87$ (s, 2H), 4.77 (s, 2H), 3.20 (s, 3H), 3.07 (s, $3 \mathrm{H}) ;{ }^{13} \mathrm{C} \mathrm{NMR}\left(100 \mathrm{MHz}, \mathrm{CDCl}_{3}\right) \delta: 164.7,163.6,139.2$, 138.9, 130.9, 121.7, 119.2, 118.8, 67.1, 43.0, 36.0, 35.5. HRMS calcd for $\mathrm{C}_{12} \mathrm{H}_{14} \mathrm{~N}_{3} \mathrm{O}_{5}\left[\mathrm{M}+\mathrm{H}^{+}\right]$: 280.0933; found 
280.0938.

$\mathrm{N}, \mathrm{N}$-二甲基-2-(8-甲酰基-2 $\mathrm{H}$-1,4-苯并噁嗪-3-酮) 乙酰胺 (2d): 白色固体, 产率 75\%. m.p. 197.2 $202.7{ }^{\circ} \mathrm{C} ;{ }^{1} \mathrm{H}$ NMR $\left(400 \mathrm{MHz}, \mathrm{CDCl}_{3}\right) \delta: 10.43(\mathrm{~s}, 1 \mathrm{H})$, 7.53 (dd, $J=7.8,1.4 \mathrm{~Hz}, 1 \mathrm{H}), 7.07$ (s, $1 \mathrm{H}), 6.98$ (d, $J=1.4$ $\mathrm{Hz}, 1 \mathrm{H}), 4.82(\mathrm{~s}, 2 \mathrm{H}), 4.72(\mathrm{~s}, 2 \mathrm{H}), 3.15(\mathrm{~s}, 3 \mathrm{H}), 3.01$ (s, $3 \mathrm{H}) ;{ }^{13} \mathrm{C} \mathrm{NMR}\left(100 \mathrm{MHz}, \mathrm{CDCl}_{3}\right) \delta: 187.8,164.9,163.8$, $147.5,129.5,124.5,122.2,121.9,119.9,67.1,42.8,36.0$, 35.5. HRMS calcd for $\mathrm{C}_{13} \mathrm{H}_{15} \mathrm{~N}_{2} \mathrm{O}_{4}\left[\mathrm{M}+\mathrm{H}^{+}\right]$: 263.1032; found 263.1024 .

$N, N$-二甲基-2-(8-甲氧基- $2 H$-1,4-苯并噁嗪-3-酮) 乙酰胺 (2e): 白色固体, 产率 67\%. m.p. 101.3 $102.7{ }^{\circ} \mathrm{C} ;{ }^{1} \mathrm{H}$ NMR $\left(400 \mathrm{MHz}, \mathrm{CDCl}_{3}\right) \delta: 6.91$ (d, $J=8.3$ $\mathrm{Hz}, 1 \mathrm{H}), 6.66(\mathrm{~d}, J=8.1 \mathrm{~Hz}, 1 \mathrm{H}), 6.41(\mathrm{~d}, J=8.0 \mathrm{~Hz}, 1 \mathrm{H})$, 4.72 (s, 2H), 4.67 (s, 2H), 3.88 (s, 3H), 3.12 (s, 3H), 2.99 (s, 3H); ${ }^{13} \mathrm{C}$ NMR $\left(100 \mathrm{MHz}, \mathrm{CDCl}_{3}\right) \delta: 165.4,164.7$, 148.4, 134.0, 129.6, 122.1, 107.1, 107.0, 67.3, 55. 8, 43.0, 35.9, 35.4. HRMS calcd for $\mathrm{C}_{13} \mathrm{H}_{16} \mathrm{~N}_{2} \mathrm{O}_{4} \mathrm{Na}\left[\mathrm{M}+\mathrm{Na}^{+}\right]$: 287.1008; found 287.1018.

$N, N$-二甲基-2-(6-乙氧酰基- $2 H$ - 1 ,4-苯并噁嗪-3酮)乙酰胺(2f): 白色固体, 产率 70\%. m.p. 136.9 $138.5{ }^{\circ} \mathrm{C} ;{ }^{1} \mathrm{H}$ NMR $\left(400 \mathrm{MHz}, \mathrm{CDCl}_{3}\right) \delta: 6.98(\mathrm{~d}, J=8.7$ $\mathrm{Hz}, 1 \mathrm{H}), 6.76 \sim 6.68(\mathrm{~m}, 1 \mathrm{H}), 6.55(\mathrm{~d}, J=2.5 \mathrm{~Hz}, 1 \mathrm{H})$, 4.66 (s, 2H), 4.64 (s, 2H), 3.12 (s, 3H), 3.00 (s, 3H), 2.27 $(\mathrm{s}, 3 \mathrm{H}) ;{ }^{13} \mathrm{C}$ NMR $\left(100 \mathrm{MHz}, \mathrm{CDCl}_{3}\right) \delta: 169.1,165.2$, 164.6, 145.2, 142.3 , 129.4, 116.8, 116.1, 108.5, 67.1, 42.9, 36.0, 35.4, 20.6. HRMS calcd for $\mathrm{C}_{14} \mathrm{H}_{16} \mathrm{~N}_{2} \mathrm{O}_{5} \mathrm{Na}[\mathrm{M}+$ $\mathrm{Na}^{+}$]: 315.0957; found 315.0961.

$\mathrm{N}, \mathrm{N}$-二甲基-2-(6- 甲氧基- $2 \mathrm{H}-1,4$ - 苯并噁嗪-3酮)-2-乙酰氧基乙酰胺 (2g): 白色固体, 产率 $52 \%$. m.p. $157.3 \sim 159.1{ }^{\circ} \mathrm{C} ;{ }^{1} \mathrm{H}$ NMR $\left(400 \mathrm{MHz}, \mathrm{CDCl}_{3}\right) \delta$ : $7.01(\mathrm{~d}, J=8.8 \mathrm{~Hz}, 1 \mathrm{H}), 6.68(\mathrm{~s}, 1 \mathrm{H}), 6.61 \sim 6.55(\mathrm{~m}, 1 \mathrm{H})$, $6.45(\mathrm{~d}, J=2.7 \mathrm{~Hz}, 1 \mathrm{H}), 4.93(\mathrm{~d}, J=16.4 \mathrm{~Hz}, 1 \mathrm{H}), 4.54$ (d, $J=16.4 \mathrm{~Hz}, 1 \mathrm{H}), 3.81 \sim 3.76(\mathrm{~m}, 3 \mathrm{H}), 3.14(\mathrm{~d}, J=5.8 \mathrm{~Hz}$, $3 \mathrm{H}), 3.02(\mathrm{~s}, 3 \mathrm{H}), 2.12 \sim 2.06(\mathrm{~m}, 3 \mathrm{H}) ;{ }^{13} \mathrm{C}$ NMR $(100$ $\left.\mathrm{MHz}, \mathrm{CDCl}_{3}\right) \delta: 168.6,164.7,159.6,155.4,134.5,127.8$, $117.8,107.2,102.1,87.2,55.3,42.9,35.9,35.5,20.3$. HRMS Calcd for $\mathrm{C}_{15} \mathrm{H}_{18} \mathrm{~N}_{2} \mathrm{O}_{6} \mathrm{Na}\left[\mathrm{M}+\mathrm{Na}^{+}\right]$: 345.1063; found 345.1054 .

$N, N$-二甲基-2-(6-氟- $2 H$-1,4-苯并噁嗪-3-酮) 乙酰 胺(2h): 白色固体, 产率 $78 \%$. m.p. $160.8 \sim 162.5{ }^{\circ} \mathrm{C}$; ${ }^{1} \mathrm{H}$ NMR $\left(400 \mathrm{MHz}, \mathrm{CDCl}_{3}\right) \delta: 6.92(\mathrm{dd}, J=8.8,5.1 \mathrm{~Hz}$, $1 \mathrm{H}), 6.67$ (td, $J=8.5,2.8 \mathrm{~Hz}, 1 \mathrm{H}), 6.48(\mathrm{dd}, J=9.5,2.8$ $\mathrm{Hz}, 1 \mathrm{H}), 4.64$ (s, 2H), 4.63 (s, 2H), 3.13 (s, 3H), 3.00 (s, $3 \mathrm{H}) ;{ }^{13} \mathrm{C} \mathrm{NMR}\left(100 \mathrm{MHz}, \mathrm{CDCl}_{3}\right) \delta: 164.9,164.7,159.0$,
156.6, 140.7, 129. 8, 129.7, 117.1, 117.0, 109.4, 109.2, $102.5,102.3,67.1,42.8,35.9,35.4$. HRMS calcd for $\mathrm{C}_{12} \mathrm{H}_{13} \mathrm{FN}_{2} \mathrm{O}_{3} \mathrm{Na}\left[\mathrm{M}+\mathrm{Na}^{+}\right]$275.0808; found 275.0816.

$\mathrm{N}, \mathrm{N}$-二甲基-2-(6-甲基- $2 \mathrm{H}$-1,4-苯并噁嗪-3-酮)乙 酰胺 (2i): 白色固体，产率 82\%. m.p. 136.3 $138.4{ }^{\circ} \mathrm{C} ;{ }^{1} \mathrm{H}$ NMR $\left(400 \mathrm{MHz}, \mathrm{CDCl}_{3}\right) \delta: 6.87(\mathrm{~d}, J=8.1$ $\mathrm{Hz}, 1 \mathrm{H}), 6.77$ (dd, $J=8.1,1.1 \mathrm{~Hz}, 1 \mathrm{H}), 6.55(\mathrm{~s}, 1 \mathrm{H}), 4.66$ (s, 2H), $4.63(\mathrm{~s}, 2 \mathrm{H}), 3.14(\mathrm{~s}, 3 \mathrm{H}), 3.02(\mathrm{~s}, 3 \mathrm{H}), 2.28(\mathrm{~s}$, $3 \mathrm{H}) ;{ }^{13} \mathrm{C}$ NMR $\left(100 \mathrm{MHz}, \mathrm{CDCl}_{3}\right) \delta: 165.5,164.9,142.5$, 132.0, 128.4, 123.9, 116.1, 115.0, 67.2, 42.8, 36.0, 35.4, 20.6. HRMS calcd for $\mathrm{C}_{13} \mathrm{H}_{17} \mathrm{~N}_{2} \mathrm{O}_{3}\left[\mathrm{M}+\mathrm{H}^{+}\right]$: 249.1239; cound 249.1243 .

$\mathrm{N}, \mathrm{N}$-二甲基-2-(7-氯-2H-1,4-苯并噁嗪-3-酮) 乙酰 胺(2j): 白色固体, 产率 $60 \%$. m.p. $154.0 \sim 157.6{ }^{\circ} \mathrm{C}$; ${ }^{1} \mathrm{H}$ NMR $\left(400 \mathrm{MHz}, \mathrm{CDCl}_{3}\right) \delta: 7.00(\mathrm{~d}, J=2.2 \mathrm{~Hz}, 1 \mathrm{H})$, $6.95(\mathrm{dd}, J=8.6,2.2 \mathrm{~Hz}, 1 \mathrm{H}), 6.67(\mathrm{~d}, J=8.6 \mathrm{~Hz}, 1 \mathrm{H})$, $4.67(\mathrm{~s}, 2 \mathrm{H}), 4.66(\mathrm{~s}, 2 \mathrm{H}), 3.13$ (s, 3H), $3.00(\mathrm{~s}, 3 \mathrm{H}) ;{ }^{13} \mathrm{C}$ NMR (100 MHz, $\left.\mathrm{CDCl}_{3}\right) \delta: 165.1,164.1,145.2,128.3$, 127.5, 122.3, 116. 9, 115.3, 67.0, 42.7, 36.0, 35.4. HRMS calcd for $\mathrm{C}_{12} \mathrm{H}_{13} \mathrm{ClN}_{2} \mathrm{O}_{3} \mathrm{Na}\left[\mathrm{M}+\mathrm{Na}^{+}\right]$: 291.0512; found 291.0500 .

$\mathrm{N}, \mathrm{N}$-二甲基-2-(7-氯-2-甲基-2 $\mathrm{H}$-1,4-苯并噁嗪-3酮)乙酰胺(2k): 白色固体, 产率 95\%. m.p. 137.0 $139.1{ }^{\circ} \mathrm{C} ;{ }^{1} \mathrm{H}$ NMR $\left(400 \mathrm{MHz}, \mathrm{CDCl}_{3}\right) \delta: 7.00(\mathrm{~d}, J=2.0$ $\mathrm{Hz}, 1 \mathrm{H}), 6.97 \sim 6.89(\mathrm{~m}, 1 \mathrm{H}), 6.65(\mathrm{~d}, J=8.6 \mathrm{~Hz}, 1 \mathrm{H})$, $4.74 \sim 4.69(\mathrm{~m}, 1 \mathrm{H}), 4.69 \sim 4.49(\mathrm{~d}, 2 \mathrm{H}), 3.12(\mathrm{~s}, 3 \mathrm{H}), 3.00$ (s, 3H), $1.57(\mathrm{~d}, J=6.8 \mathrm{~Hz}, 3 \mathrm{H}) ;{ }^{13} \mathrm{C}$ NMR $(100 \mathrm{MHz}$, $\left.\mathrm{CDCl}_{3}\right) \delta$ : 166.6, 165.3, 144.4, 128.2, 127.8, 122.2, 117.2, 115.0, 73.2, 42.9, 35.9, 35.4, 15.7. HRMS calcd for $\mathrm{C}_{13} \mathrm{H}_{15} \mathrm{ClN}_{2} \mathrm{O}_{3} \mathrm{Na}\left[\mathrm{M}+\mathrm{Na}^{+}\right]$: 305.0669; found 305.0662.

$\mathrm{N}, \mathrm{N}$-二甲基-2-(2-甲基-2H-1,4-苯并噁嗪-3-酮)乙 酰胺(2I): 白色固体, 产率 $72 \%$. m.p. $79.7 \sim 81.2{ }^{\circ} \mathrm{C}$; ${ }^{1} \mathrm{H}$ NMR $\left(400 \mathrm{MHz}, \mathrm{CDCl}_{3}\right) \delta: 6.98(\mathrm{~m}, J=7.0,3.6 \mathrm{~Hz}$, $3 \mathrm{H}), 6.75 \sim 6.70(\mathrm{~m}, 1 \mathrm{H}), 4.73(\mathrm{~d}, J=13.3 \mathrm{~Hz}, 1 \mathrm{H}), 4.71$ (d, $J=3.6 \mathrm{~Hz}, 1 \mathrm{H}), 4.60(\mathrm{~d}, J=16.5 \mathrm{~Hz}, 1 \mathrm{H}), 3.13(\mathrm{~s}, 3 \mathrm{H})$, 3.00 (s, 3H), 1.58 (d, $J=6.8 \mathrm{~Hz}, 3 \mathrm{H}) ;{ }^{13} \mathrm{C}$ NMR $(100 \mathrm{MHz}$, $\left.\mathrm{CDCl}_{3}\right) \delta: 167.2,165.6,143.9,128.9,123.4,122.3,116.8$, 114.2, 73.0, 42.9, 35.9, 35.4, 15.7. HRMS calcd for $\mathrm{C}_{13} \mathrm{H}_{16} \mathrm{~N}_{2} \mathrm{O}_{3} \mathrm{Na}\left[\mathrm{M}+\mathrm{Na}^{+}\right]: 271.1059$; found 271.1070.

$\mathrm{N}, \mathrm{N}$-二甲基-2-(6-氯-8-甲基- $2 \mathrm{H}$-1,4-苯并噁嗪-3酮)乙酰胺 (2m): 白色固体, 产率 $92 \%$. m.p. 145.7 $147.2{ }^{\circ} \mathrm{C} ;{ }^{1} \mathrm{H}$ NMR $\left(400 \mathrm{MHz}, \mathrm{CDCl}_{3}\right) \delta: 6.87 \sim 6.82(\mathrm{~m}$, $1 \mathrm{H}), 6.56(\mathrm{~d}, J=2.2 \mathrm{~Hz}, 1 \mathrm{H}), 4.67$ (s, 2H), $4.63(\mathrm{~s}, 2 \mathrm{H})$, $3.13(\mathrm{~s}, 3 \mathrm{H}), 3.01(\mathrm{~s}, 3 \mathrm{H}), 2.21(\mathrm{~s}, 3 \mathrm{H}) ;{ }^{13} \mathrm{C}$ NMR $(100$ $\left.\mathrm{MHz}, \mathrm{CDCl}_{3}\right) \delta: 164.9,164.6,141.4,129.4,127.6,126.5$, 
124.7, 112.3, 67.0, 42.9, 35.9, 35.4, 15.0. HRMS calcd for $\mathrm{C}_{12} \mathrm{H}_{15} \mathrm{ClN}_{2} \mathrm{O}_{3} \mathrm{Na}\left[\mathrm{M}+\mathrm{Na}^{+}\right]$: 291.0512; found 291.0500 .

$N, N$-二甲基-2-(2H-[2,3- $b]-1,4$-菜并噁嗪-3-酮)乙酰 胺(2n): 白色固体, 产率 49\%. m.p. 199.9 202.6 ${ }^{\circ} \mathrm{C}$; ${ }^{1} \mathrm{H}$ NMR (400 MHz, $\left.\mathrm{CDCl}_{3}\right) \delta: 7.69(\mathrm{t}, J=8.1 \mathrm{~Hz}, 2 \mathrm{H})$, $7.36(\mathrm{dd}, J=11.2,5.4 \mathrm{~Hz}, 3 \mathrm{H}), 7.11(\mathrm{~s}, 1 \mathrm{H}), 4.80$ (s, 2H), $4.75(\mathrm{~s}, 2 \mathrm{H}), 3.19(\mathrm{~s}, 3 \mathrm{H}), 3.05(\mathrm{~s}, 3 \mathrm{H}) ;{ }^{13} \mathrm{C}$ NMR $(100$ $\left.\mathrm{MHz}, \mathrm{CDCl}_{3}\right) \delta: 165.2,144.0,129.9,129.4,129.1,126.8$, $126.1,125.1,124.5,112.4,111.4,67.2,43.3,36.0,35.5$. HRMS calcd for $\mathrm{C}_{16} \mathrm{H}_{16} \mathrm{~N}_{2} \mathrm{O}_{3} \mathrm{Na}\left[\mathrm{M}+\mathrm{Na}^{+}\right]$: 307.1059 ; found 307.1049 .

辅助材料(Supporting Information) 化合物 $1 \mathrm{a} \sim 1 \mathrm{n}$ 的 合成方法, 化合物 $1 \mathrm{a} \sim \mathbf{1 n}$ 的 ${ }^{1} \mathrm{H} \mathrm{NMR}$ 和 ${ }^{13} \mathrm{C}$ NMR 谱图, 化合物 $\mathbf{2 a} \sim 2 \mathbf{n}$ 的 ${ }^{1} \mathrm{H}$ NMR 和 ${ }^{13} \mathrm{C} \mathrm{NMR}$ 谱图. 这些材料 可以免费从本刊网站(http://sioc-journal.cn/)上下载.

\section{Referenes}

[1] Sawada, Y.; Yanai, T.; Nakagawa, H.; Tsukamoto, Y.; Yokoi, S.; Yanagi, M.; Toya, T.; Sugizaki, H.; Kato, Y.; Shirakura, H.; Watanabe, T.; Yajima, Y.; Kodama, S.; Masui, A. Pest Manage. Sci. 2003, 59, 36.

[2] (a) Anderluh, P. S.; Anderluh, M.; Ilaš, J.; Mravljak, J.; Dolenc, M. S.; Stegnar, M.; Kikelj, D. J. Med. Chem. 2005, 48, 3110. (b) Rajitha, C.; Dubey, P. K.; Sunku, V.; Piedrafita, F. J.; Veeramaneni, V. R.; Pal, M. Eur. J. Med. Chem. 2011, 46, 4887.

[3] Macchiarulo, A.; Costantino, G.; Daniele Fringuelli, D.; Vecchiarelli, A.; Schiaffella, F.; Fringuelli, R. Biol. Med. Chem. 2002, 10, 3415.

[4] (a) Feng, R.; Houseman, J. G.; Downe, A. E. R. Pestic. Biol. Physiol. 1992, 42, 203.

(b) Campos, F.; Atkinson, J.; Arnason, J. T.; Philogéne, B. J. R.; Morand, P.; Werstiuk N. H.; Timmins, G. J. Chem. Ecol. 1988, 14, 989.

(c) Campos, F.; Atkinson, J.; Arnason, J. T.; Philogéne, B. J. R.; Morand, P.; Werstiuk N. H.; Timmins, G. J. Chem. Ecol. 1989, 15, 1989.

[5] Huang, M. Z.; Huang, K. L.; Ren, Y. G.; Lei, M. X.; Huang, L.; Hou, Z. K.; Ou, X. M. J. Agric. Food. Chem. 2005, 53, 7908.

[6] Holly, F. W.; Cope, A. C. J. Am. Chem. Soc. 1944, 66, 1875.

[7] (a) Russell, V. M.; Koenig, J. L.; Low, H. Y.; Ishida, H. J. Appl. Polym. Sci. 1998, 70, 1413.

(b) Shen, S. B.; Ishida, H. J. Polym. Sci., Part B: Polym. Phys. $1999,37,3257$.

(c) Takeichi, T.; Kawauchi, T.; Agag, T. Polym. J. 2008, 40, 1121.

(d) Xiang, H.; Ling, H.; Wang, J.; Song, L.; Gu, Y. Polym. Compos. $\mathbf{2 0 0 5}, 26,563$

[8] Hamilton, R. H.; Bandurski, R. S. Cereal Chem. 1962, 39, 107.

[9] (a) Zuniga, G. E.; Argandona, V. H.; Niemeyer, H. M.; Corcuera, L. J. Phytochemistry 1983, 22, 2665.

(b) Erich, G.; Wolfgang, E.; Bacher, A.; Frey, M.; Gierl, A. Phytochemistry 1997, 45, 715 .

[10] (a) Rajachandrashekar, V.; Vineel, B. G.; Venkataiah S. Pharm. Chem. 2014, 6, 7 .

(b) Rajitha, C.; Dubey, P. K.; Dubey, P. K.; Sunku, V.; Veeramaneni, V. R.; Pal, M. J. Heterocycl. Chem. 2013, 50, 630.

(c) Armitage, M.; Bret, G.; Choudary, B. M.; Kingswood, M.; Loft, M.; Moore, S.; Smith,S.; Urquhart, M. W. J. Org. Process Res. Dev. 2012, 16, 1626.

(d)Macías, F. A.; Marín, D.; Bastidas, A.O.; Chinchilla, D.;Simonet, A. M.; Molinillo, J. M. G. J. Agric. Food Chem. 2006, 54, 991.

(e) Ramesh, C.; Raju, B. R.; Kavala, V.; Kuo, C. W.; Yao, C. F. Tetrahedron 2011, 67, 1187 .

(f) Gangloff, A. R.; Brown, J.; Jong, R.; Dougan, D. R.; Grimshaw, C. E.; Hixon, M.; Jennings, A.; Kamran, R.; Kiryanov, A.; Connell, S.; Taylor, E. J. Biol. Med. Chem. Lett. 2013, 23, 4501.

(g) Sams, A. G. Hentzer, M.; Mikkelsen, G. K.; Larsen, K.; Bundgaard, C.; Plath, N.; Christoffersen, C. T.; Andersen, B. B. J. Med. Chem. 2010, 53, 6386.

[11] (a) Sharifi, A.; Barazandeh, M.; Abaee, M. S.; Mirzaei, M. Terahedron Lett. 2010, 51, 1852.

(b) Brahma, K.; Das, B.; Chowdhury, C. Terahedron 2014, 70, 5863.

(c) Konda, S.; Raparthi, S.; Bhaskar, K.; Munaganti, R. K.; Guguloth, V.; Nagarapu, L.; Akkewar, D. M. Bioorg. Med. Chem. Lett. 2015, 25, 1643.

(d) Borate, H. B.; Maujan, S. R.; Sawargave, S. P.; Chandavarkar, M. A.; Vaiude, S. R.; Joshi, V. A.; Wakharkar, R. D.; Ramkilyer. Bioorg. Med. Chem. Lett. 2010, 20, 722.

[12] Zuo, H.; Li, Z. B.; Ren, F. K.; Jalck, J. R.; Meng, L. J.; Ahn, C.; Shin, D. S. Terahedron Lett. 2008, 64, 9669.

[13] Chen, D. B.; Shen, G. D.; Bao, W. L. Org. Biomol. Chem. 2009, 7, 4067.

[14] Min, E. Z.; Wu, W. Green Chemistry and Chemical Engineering, Chemical Industry Press, Beijing, 2000, pp. 22 24 (in Chinese). (闵恩泽, 吴嵬, 绿色化学与化工, 北京, 化学化工出版社, 北 京, 2000, pp. 22 24.)

[15] (a) Zaitsev, V. G.; Shabashov, D.; Daugulis, O. J. Am. Chem. Soc. 2005, 127, 13154.

(b) Shabashov, D.; Daugulis, O. J. Am. Chem. Soc. 2010, 132, 3965.

[16] (a) Kim, J.; Sim, M.; Kim, N.; Hong, S. Chem. Sci. 2015, 6, 3611. (b) Castro, L. C. M.; Chatani, N. Chem. Eur. J. 2014, 20, 4548.

[17] Chen, G.; Shigenari, T.; Jain, P.; Zhang, Z. P.; Jin, Z.; He, J.; Li, S. H.; Mapelli, C.; Miller, M. M.; Poss, M. A.; Scola, P. M.; Yeung, K. S.; Yu, J. Q. J. Am. Chem. Soc. 2015, 137, 3338.

[18] Guan, M. Y.; Pang. Y. B.; Zhang, J. Y.; Zhao, Y. S. Chem. Commun. 2016, $52,7043$.

[19] He, G.; Lu, C. X.; Zhao, Y. S.; Nack, W. A.; Chen, G. Org. Lett. 2012, 14, 2944. 